\title{
Efeito dos Ácidos Graxos Ômega-3 sobre o Relaxamento- Dependente do Endotélio em Coelhos Hipercolesterolêmicos
}

\author{
Paulo A fonso Ribeiro Jorge, Lidia Cruz N eyra, Regina Michico O zaki, Eros de Almeida \\ Campinas, SP
}

Objetivo - Estudar o efeito dos ácidos graxos ômega3 sobre o relaxamento-dependente do endotélio, o colesterol plasmático, as $L D L, V L D L, H D L$, triglicérides e a peroxidação lipídica das partículas de LDL-nativas, oxidadas e da parede arterial.

Métodos - Coelhos da raça Nova Zelândia foram submetidos a dieta enriquecida com colesterol $(0,5 \%) e$ gordura de coco (2\%), por 30 dias e separados em grupo hipercolesterolemia $(H)$ e ômega-3 (O-3), sendo administrado ao $O-3$ ácidos graxos ômega-3 na dose de 300mg/ $\mathrm{kg} /$ dia, durante 15 dias, através de gavagem. O colesterol plasmático, triglicérides, LDL-colesterol, VLDL e HDLcolesterol foram medidos através de kits enzimáticos e os resultados expressos em $\mathrm{mg} / \mathrm{dl}$. As LDL foram obtidas por ultracentrifugação e oxidadas através da exposição ao $\mathrm{Cu}^{++}$. A peroxidação lipídica das $L D L$ e da parede da aorta foi mensurada pela dosagem do malondialdeido (MDA). A função endotelial foi avaliada por curvas de concentração-efeito obtidas pela acetilcolina e nitroprussiato, após contração com norepinefrina.

Resultados - Houve aumento do colesterol plasmático e das VLDL, sem interferência nos níveis de LDL e $H D L$, no O-3. Observou-se redução significante dos triglicérides. Verificou-se aumento significante do teor de $M D A$ nas $L D L$-nativas e oxidadas, assim como na parede arterial. O relaxamento-dependente do endotélio foi significativamente menor no $O-3$.

Conclusão - A administração de ácidos graxos ômega-3 na dosagem de 300/mg/kg/dia, a coelhos hipercolesterolêmicos aumentou o colesterol e as VLDL plasmáticas, enquanto reduziu os triglicérides. $O$ relaxamentodependente do endotélio foi menor que no grupo $\mathrm{H}$.

Palavras-chave: relaxamento-dependente do endotélio, ácidos graxos ômega-3, hipercolesterolemia, malondialdeido

\section{Effects of Omega-3 Fatty Acids on Endothelium-Dependent Relaxation in Hypercholesterolemic Rabbits}

Purpose - To study the effect of omega-3 fatty acid on endothelium-dependent relaxation, total plasma cholesterol, LDL-cholesterol, HDL-cholesterol and triglycerides levels as well as, the malondialdehyde (MDA) content of the LDL particles and arterial wall.

Methods - Fourteen male rabbits were randomly assigned to hypercholesterolemic and omega-3 groups. The dose of omega-3 fatty acid utilized was $300 \mathrm{~g} / \mathrm{kg} /$ day during 15 days. All rabbits were fed a diet supplemented with cholesterol ( $0.5 \%)$ and coconut oil (2\%) for four weeks. At the end of the experiment the animals were killed and the aorta removed formeasurement of MDA content and the endothelium-dependent relaxation studies. Total plasma cholesterol, VLDL-cholesterol, LDL-cholesterol, HDL-cholesterol and triglycerides was measured by enzymatic kits. MDA was also measured in natives and oxidized LDL and arterial wall.

Results - Cholesterol and VLDL-cholesterol were increased significantly in the omega-3 treated animals. The triglyceride level was significantly reduced $(p<0.05)$. The MDA content was increased in the LDL particles and in the arterial wall $(p<0.05)$. Endothelium-dependent relaxation was significantly reduced $(p<0.05)$.

Conclusion - Omega-3 fatty acid impairs the endothelium-dependent relaxation when administered to cholesterolfed rabbits, increases the cholesterol and reduces the triglycerides plasma levels. The lipid peroxidation of the LDL particles and arterial wall was increased.

Key-words: endothelium-dependent relaxation, omega-3 fatty acid, hypercholesterolemia

Arq Bras Cardiol, volume 69 (n' 1), 13-18, 1997

Núcleo de Medicina e Cirurgia Experimental - UNICAMP - Campinas Correspondência: Paulo Afonso Ribeiro Jorge - Rua Guilherme da Silva, 397/31 - 13025-070 - Campinas, SP

Recebido para publicação em 20/2/97

Aceito 7/5/97
A aterosclerose coronária é responsável pela alta mortalidade de indivíduos após os 40 anos de idade, representando o infarto do miocárdio, a maior causa de morte nesse grupo etário ${ }^{1}$. Tem-se verificado, de outro lado, que a redução do colesterol plasmático, através de drogas hipoli- 
pemiantes é capaz de reduzir em até $50 \%$ a ocorrência de eventos, como infarto fatal e não fatal ${ }^{2}$. Estudos angiográficos bem conduzidos mostraram que a redução dos eventos coronários ocorreu sem redução significativa do diâmetro da obstrução do vaso pela placa de aterosclerose ${ }^{3}$. Diante destes achados, tem-se imputado à redução dos eventos coronários, após a redução do colesterol plasmático, a estabilidade da placa de aterosclerose e a preservação da função endotelial ${ }^{4}$. Assim, numerosos estudos têm procurado verificar o efeito de diferentes substâncias, na ação protetora sobre a função endotelial.

O endotélio vascular é responsável pela produção do $\mathrm{NO}^{\circ}$ e PG12, substâncias vasodilatadoras e que também interagem na adesão e agregação das plaquetas, aderência de neutrófilos etc, evitando o vasoespasmo e a trombose coronária, principais mecanismos envolvidos nas síndromes coronárias agudas 5,6 .

Em trabalho inicial, demonstramos que a hipercolesterolemia induzida em coelhos da raça Nova Zelândia através da dieta, comprometia o relaxamento-dependente do endotélio, em tiras de aorta torácica ${ }^{7}$ e suas conclusões comprovam os resultados de trabalhos similares publicados na literatura ${ }^{8}$. Na mesma linha de pesquisa, verificamos que é possível reverter a disfunção endotelial na hipercolesterolemia, após tratamento com inibidores da HMGCoA redutase $^{7}$, também conforme literatura ${ }^{9}$. Mais recentemente, estudamos a velocidade de reversão da disfunção endotelial em coelhos hipercolesterolêmicos, administrando simvastatina e pravastatina durante os 2, 4 e 6 últimos dias do experimento ${ }^{10}$.

Considerando a eventual influência de substâncias antioxidantes na preservação da função endotelial, estudamos o efeito da vitamina E sobre o fluxo coronário dependente do endotélio, em cães hipercolesterolêmicos ${ }^{11}$. Nesse estudo constatamos, pela primeira vez, que a vitamina E reverte a disfunção endotelial na microcirculação coronária, assim como, reduz o teor do colesterol e malondialdeido (MDA) teciduais, sem influir no nível do colesterol plasmático.

Neste trabalho, nos propusemos a estudar a influência dos ácido graxos ômega-3 sobre a reversão da disfunção endotelial na hipercolesterolemia, bem como confrontar a função endotelial com o nível do colesterol total plasmático, LDL-colesterol, HDL-colesterol, triglicérides e a peroxidação lipídica das partículas de LDL e da parede arterial.

\section{Métodos}

Coelhos da raça Nova Zelândia com peso de 2,14士0,16 $\mathrm{kg}$ e idades semelhantes foram divididos de forma aleatória em dois grupos de sete animais: grupo hipercolesterolemia (H) e grupo ômega-3 (O-3). Todos os animais foram alimentados com $100 \mathrm{~g}$ de ração ao dia, acrescentada de $0,5 \%$ de colesterol e $2 \%$ de gordura de coco, durante 30 dias. Água foi oferecida ad libitum. Ácidos graxos ômega-3 (ácido eicosapentaenóico-EPA e ácido graxo docosahexanaenóico-DHA) foram administrados na dose de $300 \mathrm{mg} / \mathrm{kg} /$ dia $(\mathrm{EPA}=180 \mathrm{mg}$ e DHA=120mg), através de gavagem, nos últimos 15 dias de dieta. No último dia do experimento, uma amostra de sangue obtida por punção cardíaca foi utilizada para dosagem do colesterol plasmático, HDL-colesterol, VLDL e triglicérides, através de kits enzimáticos e analisador Beckman 700.

Anéis da aorta torácica de cada animal, com aproximadamente $5 \mathrm{~mm}$, foram limpos de tecido conectivo, cuidandose para preservar o endotélio. O endotélio foi mecanicamente removido em outros segmentos da aorta torácica dos mesmos animais. Os anéis foram suspensos em câmara de perfusão com $10 \mathrm{~mL}$ de capacidade, contendo solução de Krebs-Henseleit a pH 7,4, com a composição mmol/L: $\mathrm{NaCl}$, 113,$0 ; \mathrm{CaCl}_{2}, 1,9 ; \mathrm{NaHCO}_{3}, 25,0 ; \mathrm{MgSO}_{4}, 0,44 ; \mathrm{KH}_{2} \mathrm{PO}_{4}, 1,18$; EDTA, 0,03; glicose, 11,0 . A solução foi mantida a $37^{\circ} \mathrm{Ce}$ continuadamente aerada com uma mistura de gases contendo $95 \%$ de $\mathrm{O}_{2}$ e $5 \%$ de $\mathrm{CO}_{2}$. Os anéis foram montados em dois clips de aço, ligados a um suporte de um lado e a um transdutor de força, da marca Narco Biosystem, de outro lado. Os anéis foram distendidos a uma tensão de $1 \mathrm{~g}$, estabelecida previamente como tensão ideal, através de curvas de comprimento-tensão. Os espécimes foram perfundidos por um período de $60 \mathrm{~min}$, trocando-se a solução a cada 20min. Para prevenir a síntese de prostaglandinas os experimentos foram realizados na presença de $10 \mu \mathrm{m}$ de indometacina. Os resultados foram expressos como porcentagem de relaxamento em relação à contração com $\mathrm{NE}$.

No protocolo de experimentação, todos os anéis de aorta, com e sem endotélio, foram contraídos pela adição de norepinefrina-NE $\left(10^{-7} \mathrm{M}\right)$. Após estabilização da contração, acetilcolina-Ach foi adicionada ao banho de forma cumulativa, para alcançar concentrações de $10^{-8}$ a $10^{-5.5} \mathrm{M}$, obtendo-se as curvas de concentração-efeito. Após, a solução de Krebs-Henseleit foi substituída por solução fresca e a tensão relaxada até o valor basal. Depois de um período de $30 \mathrm{~min}$ os anéis foram contraídos com NE $\left(10^{-7} \mathrm{M}\right)$ e outras curvas de concentração-efeito obtidas com nitroprussiato$\operatorname{SNP}\left(10^{-8}\right.$ a $\left.10^{-5.5} \mathrm{M}\right)$.

A peroxidação lipídica da parede do vaso foi mensurada pelo teor de MDA, um dos produtos da peroxidação lipídica. Amostras de aorta foram homogeneizadas em ácido tricloroacético gelado (mg de tecido por ml de TCA a $10 \%$ ). Após centrifugação, uma alíquota do sobrenadante foi acrescentada a igual volume de ácido tiobarbitúrico a 0,6\% e a mistura aquecida a $100^{\circ} \mathrm{C}$ por $20 \mathrm{~min}$. A concentração de MDA foi calculada pela absorção a $532 \mathrm{~nm}$ com o uso de um coeficiente de extinção molar de $1,49 \times 10^{-5}$ e expressa em $\mathrm{nmol} / \mathrm{mg}$ de tecido x $10^{-712}$.

Amostras de sangue foram coletadas em tubos de plástico contendo apropriados volumes de solução aquosa de EDTA a 15\%, pH 7,4, para uma concentração final de $0,15 \%$ de EDTA, que serve como anticoagulante e antioxidante. O sangue foi centrifugado a $1000 \mathrm{rpm}$ por $10 \mathrm{~min}$. $\mathrm{O}$ sobrenadante, à temperatura de $10^{\circ} \mathrm{C}$, foi centrifugado a 1000 rpm por $5 \mathrm{~min}$, seguida de outra centrifugação de $15000 \mathrm{rpm}$ por $10 \mathrm{~min}$. Este procedimento remove todos os resíduos celulares e o plasma obtido é límpido. 
O método de isolamento das LDL consiste em dois passos seqüenciais de ultracentrifugação: $1^{\circ}$ ) o plasma (>4ml) é centrifugado a $40000 \mathrm{rpm}$ por $18 \mathrm{~h}$ (ultracentrífuga Beckman, mod.L-8) para separar as partículas de quilomícrons e VLDL (d>1063), e com uma pipeta Pasteur estas partículas são removidas; $2^{\circ}$ ) a densidade do remanescente é ajustada a 1063, através de $\mathrm{KBr}$ sólido. O isolamento das LDL ( $D=10063$ a 1063) faz-se por centrifugação a 40000rpm durante 20h. As LDL são coletadas com pipeta de ponta fina para sua caracterização e oxidação ${ }^{13}$.

A caracterização química das LDL está baseada na determinação do LDL-colesterol total usando kits enzimáticos da Boehringer Manhein-Germany. A proteína foi quantificada pelo método de Lowry e $\mathrm{col}^{14}$.

O EDTA é removido das LDL imediatamente após a ultracentrifugação mediante diálise contra tampão fosfato a $\mathrm{pH}$ 7,4,por $24 \mathrm{~h}$. A oxidação das LDLérealizada mediante aincuba-

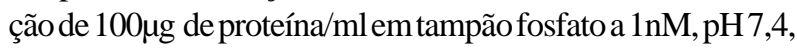
em presença de sulfato de cobre $5 \mathrm{uM} \mathrm{a} 37^{\circ} \mathrm{C}$ por $24 \mathrm{~h}^{15}$.

A peroxidação das LDL consiste em colocar em um tubo de ensaio $100 \mu \mathrm{g}$ de proteína-LDL, completar a um volume de $500 \mu \mathrm{L}$, adicionar $1 \mathrm{~mL}$ de ácido tiobarbitúrico $0,67 \%$ e $0,3 \mathrm{~mL}$ de ácido tricloroacético a $50 \%$. Após aquecimento, a $100^{\circ} \mathrm{C}$ por $30 \mathrm{~min}$, o teor de TBARS é determinado a $532 \mathrm{~nm}$ de absorvância. Usa-se como padrão o tetrametoxipropilTMP, como equivalente a concentração de MDA ${ }^{16}$, com resultados expressos em nmol/mg de proteína.

O colesterol foi obtido de Aldrich Chemical Co, Gillingham, Dorset, UK. Noradrenalina-bitartarato, acetilcolina-cloridato, nitroprussiato de sódio, 2-ácido tiobarbitúrico, hidroxitolueno e indometacina obtidos de SigmaChemical Co, Poole, UK e todas as drogas da solução de Krebs-Henseleit obtidas de BHD Lab Supplies, Poole, UK.

Os dados são apresentados como média e desvio padrão da média. Os valores do colesterol total no plasma, LDL-colesterol, HDL-colesterol, VLDL, triglicérides, teor de MDA nas LDL nativas e oxidadas e na parede arterial foram comparadas por método estatístico, não paramétrico, de Kruskal-Wallis e Mann-Whitney ${ }^{17}$. As curvas de relaxamento foram comparadas por análise de co-variância ${ }^{18}$. Valores menores que 0,05 foram considerados significantes.

\section{Resultados}

Não se observou diferença estatisticamente significante em relação aos pesos dos animais, que no final do experimento foram de $2,98 \pm 0,16$ para o grupo $\mathrm{He} 2,90 \pm 0,23$ para o grupo O-3.

O perfil lipídico está expresso na figura 1. Observaramse aumento significante do colesterol total plasmático ( $21 \%)$, das VLDL (42\%) e redução dos valores dos triglicérides $(31 \%)$ no grupo $\mathrm{O}-3(\mathrm{p}<0,05)$. Não ocorreram variações significantes para os valores do LDL-colesterol e HDLcolesterol plasmáticos. O teor de MDA aumentou de forma significante nas LDL nativas (37\%), nas LDL oxidadas (11\%) e na parede arterial (35\%) no grupo O-3 (p<0,05) (fig. 2).

A comparação das curvas de concentração-efeito

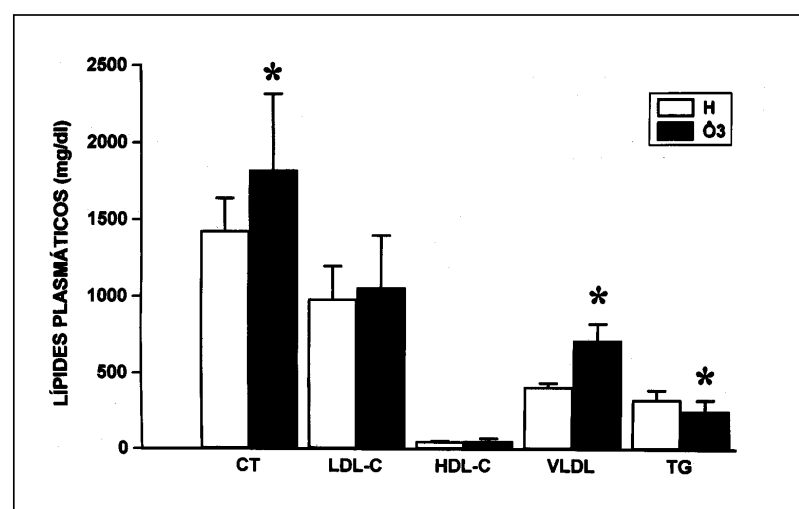

Fig. 1 - Perfil lipídico do grupo hipercolesterolemia e grupo ômega-3 Os valores são expressos em média e desvio padrão da média. H-hipercolesterolemia; O-3-ômega3; CT- colesterol total; T- triglicérides; ${ }^{*} \mathrm{p}<0,05$ em relação ao controle.

mostrou comprometimento do relaxamento-dependente do endotélio para o grupo O-3 $(\mathrm{p}<0,05)$. O relaxamento máximo foi $<30 \%$ em relação ao grupo H (fig. 3). Não se observaram diferenças significantes em relação às curvas de concentração-efeito na presença de nitroprussiato. Os anéis de aorta sem endotélio mostraram relaxamento similar ao nitroprussiato, mas sem resposta à acetilcolina. A contração inicial promovida pela norepinefrina $\left(10^{-7} \mathrm{M}\right)$ não variou significativamente nos grupos $\mathrm{He} \mathrm{O}-3$. Os valores absolutos dos parâmetros estudados estão expressos na tabela I.

Cabe destacar que todos os animais do grupo O-3 apresentaram hemopericárdio após a punção cardíaca.

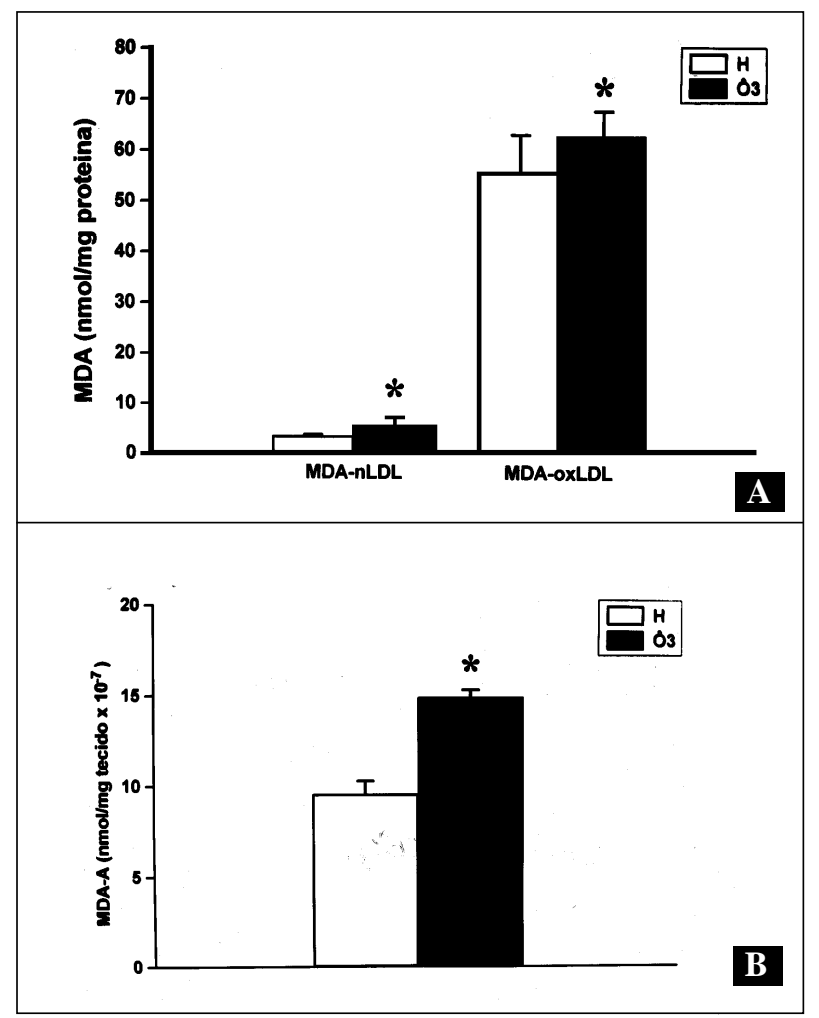

Fig. 2 - Concentração média e desvio padrão de malondialdeido (MDA) nas LDLnativas, oxidadas (A) e na parede $\operatorname{arterial}(\mathrm{B})$. * $\mathrm{p}<0,05$ em relação ao controle. 


\section{Discussão}

A possibilidade de que o consumo aumentado de ácidos graxos polinsaturados ômega-3, EPA e DHA, possa proteger contra o desenvolvimento da doença cardiovascular aterosclerótica, decorreu das observações de Bang e col ${ }^{19}$ e Dyerberg e col ${ }^{20}$, considerando a baixa prevalência de doença cardiovascular aterosclerótica em esquimós da Groenlândia que utilizam alto teor dessas substâncias na sua alimentação. Os mecanismos propostos incluem desde modificações favoráveis nos níveis de lípides plasmáticos, alterações hepáticas do metabolismo do colesterol, até redução da captação do colesterol pelo fígado. Estudos que avaliaram o efeito do EPA e DHA sobre o perfil lipídico, de um modo geral, demonstraram redução das VLDL e da trigliceridemia ${ }^{21-23}$. Entretanto, o principal efeito dos ácidos graxos ômega-3 sobre as doenças coronárias estaria na sua ação sobre a agregação plaquetária, redução na produção de TXA2 e prolongamento do tempo de sangramento ${ }^{24,25}$.

Nossos resultados mostraram que os ácidos graxos ômega-3 elevaram o colesterol plasmático e as VLDL, sem influir significativamente nos níveis das partículas de LDLcolesterol e HDL-colesterol. Os triglicérides reduziram-se de forma significante. Os valores relativos à peroxidação das LDL-nativas e oxidadas, assim como da parede arterial, mostraram-se elevados de forma significante nos animais tratados com ácidos graxos ômega-3. O relaxamento-dependente do endotélio foi menor no grupo O-3.

Os trabalhos relatados na literatura em animais de experimentação apresentam resultados conflitantes, quando se referem ao efeito dos ácidos graxos ômega-3 sobre o perfil lipídico. Em coelhos hipercolesterolêmicos, Achtani e col ${ }^{26}$ verificaram que o grupo de animais tratados com ácidos graxos ômega-3 apresentou elevação significante das BVLDL e LDL-C, assim como dos níveis de peróxidos no plasma (TBARS). Verificaram também aumento do número de células espumosas na íntima arterial. Por estes resultados os

\begin{tabular}{|c|c|c|}
\hline \multicolumn{3}{|c|}{$\begin{array}{l}\text { Tabela I - Valores médios e desvio-padrão do colesterol total } \\
\text { plasmático, LDL-colesterol, HDL-colesterol, triglicérides, MDA da } \\
\text { parede arterial, das LDL nativas e oxidativas in vitro e do } \\
\text { relaxamento máximo dependente do endotélio observados nos } \\
\text { animais controle (H) e animais tratados com ácidos graxos } \\
\text { ômega-3 }(0-3) \text {. }\end{array}$} \\
\hline & $\mathrm{H}$ & $\mathrm{O}-3$ \\
\hline $\mathrm{CT}$ & $1423,89 \pm 212,52$ & $1817,74 \pm 490,66 *$ \\
\hline LDL-C & $979,40 \pm 216,11$ & $1055,01 \pm 340,36$ \\
\hline HDL-C & $49,37 \pm 3,87$ & $53,35 \pm 17,25$ \\
\hline VLDL & $407,41 \pm 26,39$ & $709,38 \pm 109,85^{*}$ \\
\hline TG & $325,42 \pm 60,66$ & $248,42 \pm 72,33 *$ \\
\hline MDA-A & $9,56 \pm 0,73$ & $14,89 \pm 0,43^{*}$ \\
\hline MDA-nLDL & $3,63 \pm 0,30$ & $5,79 \pm 1,60 *$ \\
\hline MDA-oxLDL & $55,61 \pm 7,43$ & $62,56 \pm 5,07 *$ \\
\hline $\mathrm{T}$ & $1,75 \pm 0,20$ & $1,65 \pm 0,16$ \\
\hline R. Max & $68,82 \pm 3,21$ & $51,58 \pm 6,64 *$ \\
\hline
\end{tabular}

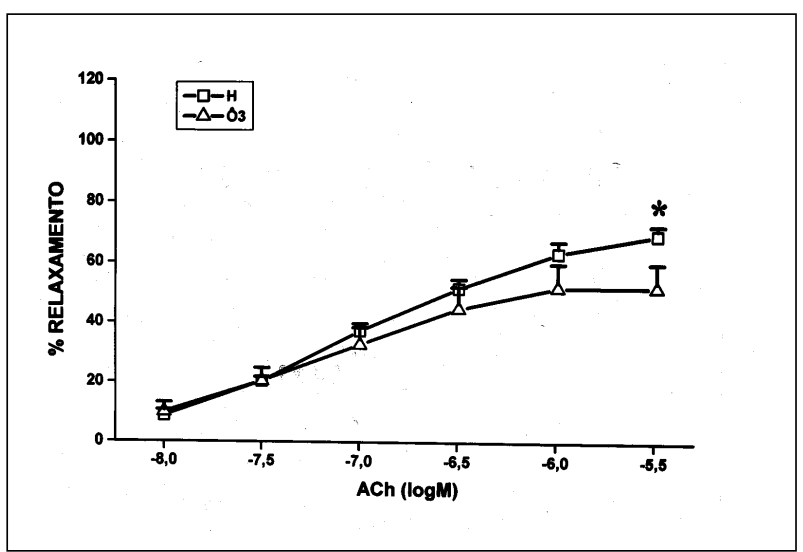

Fig. 3 - Curvas do relaxamento-dependente do endotélio em resposta à acetilcolina (ACh) após contração com norepinefrina (NE). A abcissa refere-se a concentração $(\log \mathrm{M})$ de acetilcolina e a ordenada ao relaxamento expresso como percentual da con-

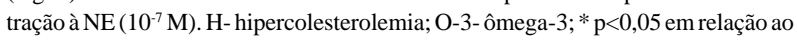
controle.

ácidos graxos ômega-3 induziriam a aterosclerose, agravando a peroxidação lipídica e elevando o colesterol plasmático. Adelstein e $\mathrm{col}^{27}$, em modelo experimental semelhante, verificaram elevação das LDL-colesterol e também dos triglicérides nos animais tratados com ácidos graxos ômega-3, embora não constatassem diferença significante com o grupo controle em relação à concentração plasmática de TBARS. Yano e $\mathrm{col}^{28}$, de outro lado, observaram em coelhos hipercolesterolêmicos redução do colesterol total nas LDL e IDL, no grupo tratado com $300 \mathrm{mg} / \mathrm{kg}$ de EPA-E por quatro semanas. Enquanto os primeiros autores não observaram diferença no número de ligações hepáticas para B-VLDL, estes últimos verificaram um significante aumento do número destes receptores para LDL, a que atribuem o efeito hipolipidêmico da droga.

Em humanos, os relatos indicam que o principal efeito dos ácidos graxos ômega-3 é a redução dos triglicérides e das VLDL ${ }^{29}$. Como as VLDL são precursoras das LDL, a redução desta lipoproteína também é relatada. Estas observações referem-se a pacientes com hipertrigliceridemia. Em indivíduos com hipercolesterolemia e níveis normais de triglicérides, os efeitos dos ácidos graxos ômega-3 sobre o LDL e HDL-colesterol são inaparentes ${ }^{30}$. Estudo recente verificou que em indivíduos com hipertrigliceridemia e também em indivíduos normais, a suplementação alimentar com ácidos graxos ômega-3 causou uma elevação paradoxal dos níveis da LDL apoproteínaB e uma diminuição dos valores dos triglicérides ${ }^{31}$.

A influência dos ácidos graxos ômega-3 sobre a função endotelial é pouco relatada na literatura. Shimokava e $\mathrm{col}^{32}$ referem aumento do relaxamento-dependente do endotélio em artérias coronárias de porco, sob dieta enriquecida com ácidos graxos ômega-3. É possível que os resultados aparentemente contraditórios, observados em relação ao perfil lipídico e a função endotelial, dependam da espécie animal estudada e das especificidades do seu metabolismo lipídico. A dose da substância utilizada pode ser 
outro fator a explicar as diferenças de resultados. No nosso trabalho, utilizamos a dose de $300 \mathrm{mg} / \mathrm{kg}$ de peso, de acordo com os trabalhos de Yano e col ${ }^{28}$ que empregaram modelo experimental semelhante.

A acentuação da peroxidação lipídica das LDL faz sentido lógico, considerando o maior número de duplas ligações nas moléculas dos ácidos graxos ômega-3. Em conseqüência, acentua-se a peroxidação da parede arterial e, por este mecanismo, somado à elevação do colesterol plasmático, compromete-se a função endotelial. De acordo com este raciocínio a administração de ácidos graxos ômega-3 deve induzir a aterogênese e agravar a aterosclerose. Entretanto, deve-se considerar o especial efeito destas substâncias sobre a agregação plaquetária, a fibrinólise e a formação das prostaciclinas, que poderá compensar os efeitos adversos sobre a aterogênese. Observações clínicas bem conduzidas mostram uma correlação inversa entre a ingestão de óleo de peixe e a mortalidade por doença coronária ${ }^{33,34}$, re- sultados que podem ser interpretados como decorrentes da ação antitrombótica da droga. Cabe verificar a longo prazo o que ocorre com a placa de aterosclerose.

Entendemos que a administração de ácidos graxos ômega-3, na dependência da dose utilizada e do animal de experimentação, aumenta a peroxidação das LDL e da parede arterial, eleva o colesterol plasmático e compromete a função endotelial, favorecendo, assim, o desenvolvimento da aterosclerose. Sua indicação ficaria reservada como agente antiplaquetário e antitrombótico e no tratamento das hipertrigliceridemias, devendo concorrer com substâncias de ação terapêutica similar, como os fibratos e a aspirina, que não apresentam efeito colateral sobre a peroxidação lipídica e a função endotelial.

\section{Agradecimentos}

À FAPESP pelo auxílio recebido.

\section{Referências}

1. Kannel EB, Castelli WR, Gordon T-Cholesterol in the prediction of atherosclerosis disease; new perspectives based on the Framingham study. Ann Int Med 1979; 90: 85-9.

2. Lipid Research Clinics Program - The Lipid Research Clinics Coronary Primary Prevention Trials Results. JAMA 1984; 251: 351-64

3. Brown G, Albers JJ, Fisher LD et al - Regression of coronary artery disease as a result of intensive lipid-lowering therapy in men with high levels of apolipoprotein B. N Engl J Med 1990; 323: 1289-98.

4. Meredith IT, Yueng AC, WeidingerFFet al-Role of impaired endothelium-dependent vasodilation in ischemic manifestation of coronary artery disease. Circulation 1993; 87(suppl V): V56-V66.

5. Moncada S, Radomski MW, Palmer RM - Endothelium-derived relaxing factor identification as nitric oxide and role in the control of vascular tone and platelet function. Biochem Pharmacol 1988; 37: 2495-501.

6. Garg UC, Hassid A - Nitric oxide generating vasodilators and 8-bromo-cyclic GMP inhibit mitogenesis and proliferation of cultured rat vascular smooth muscle cells. J Clin Invest 1990; 83: 1774-7.

7. Ribeiro Jorge PA, Ozaki RM, Metze K-Effects of simvastatin and pravastatin on endothelium-dependent relaxation in hypercholesterolemic rabbits. Exp Toxic Pathol 1994; 46: 465-9.

8. Jayakody L, Seneratne M, Thomson A, Kapagoda T - Endothelium-dependent relaxation in experimental atherosclerosis in the rabbit. Circ Res 1987; 50: 25164.

9. Osbom JA, Lento PH, Siegfried MR, Stahl GL, Fusman B, Lefer AM-Cardiovascular effects of acute hypercholesterolemia in rabbits. Reversal with lovastatin treatment. J Clin Invest 1989; 83: 466-73.

10. Ribeiro Jorge PA, Ozaki RM, AlmeidaE-Rappid reversal of endothelium-dependent dysfunction in hypercholesterolemic rabbits treated with simvastatin and pravastatin. Exp Clin Pharmacol Physiol (submitted).

11. Ribeiro Jorge PA, Ozaki RM, Almeida E, Credidio NL - Effects of vitamin E on endothelium-dependent coronary flow in hypercholesterolemic dogs. Atherosclerosis 1996; 126: 43.

12. Cassini AF, Ferrari M, Pompella A, Maellaro E, Comporti M-Lipid peroxidation and cellular damage in extrahepatic tissue of bromobenzene-intoxicated mice. Am J Pathol 1986; 123: 520-31.

13. Havel R, Eder H, Bragdon J - The distribution and chemical compositions of ultracentrifugally separated lipoproteins in humans serum. J Clin Invest 1955; 34 : 1345-53.

14. Lowry O, Rosebrough NJ, Fan A, Randell J - Protein measurement with the folin phenol reagent. J Biol Chem 1951; 193: 265-75.
15. Kleiveld H,Hak-Lemmers H, Stlenfold A, Demarck P-Improved measurement of low density lipoprotein susceptibility to copper-induced oxidation. Aplication to a short procedure for isolation low density lipoprotein. Clin Chem 1992; 38: 2066-72.

16. Parthasarathy S, Steimbrecher P, Barnett J, Witztun J, Steimberg D - Essential role of phospholipase A2 activity in endothelium cell induced modification of low density lipoprotein. Proc Natt Acad Sci USA 1985; 82: 3000-4.

17. SAS (Statistical Analysis System) SAS/TAT user's guide, version $6,4^{\text {th }}$ edit, vol 1,1990 .

18. Snedecor GW, Cochram WG-Statistical Methods. $7^{\text {th }}$ ed. Ames Iowa: Iowa State University Press, pp 385-8.

19. Bang HO, Dyerberg J, Hjorne N - The composition of food consumed by Greenland Eskimos. Acta Med Scand 1976; 200: 69-73.

20. Dyerberg J, Bang HO, Hjorne N - Fatty acid composition of the plasma lipids in Greenland Eskimos. Am J Clin Nutr 1975; 28: 958-66.

21. Harris WS, Connor WE, Goodnight Jr SH - Dietary fish oil, plasma lipids and platelets in man. Prog Lipid Res 1981; 20: 75.

22. Nestel JP, Connor EW, Reardon FM, Connor S, Wong S, Boston R - Supression by diets rich in fish oil of very low density lipoprotein production in men. J Clin Invest 1984; 74: 82.

23. Sanders TAB, Sullivan DR, Reeve J, Thompson GR - Triglyceride lowering effects of marine polyunsaturetes in patients with hypertriglyceridemia. Arteriosclerosis 1985; $5: 459$.

24. Sanders TAB, Vickers M, Haines AP-Effect on blood lipids and haemostasis of a supplement of cod-liver oil. Rich in eicopentaenoic and decosahexaenoic acids, in healthy young men. Clin Sci 1981; 61: 317.

25. Saynor R, VerelD, Gillot T - The long-term effect of dietary supplementation with fish lipid concentrate on serum lipids, bleending time, platelets and angina. Atherosclerosis 1984; 50: 3 .

26. Achtani C, Awtade A, Vasisht S, Srivastava LM-Effect of max EPA (fish oil) on lipoprotein and its receptors in hypercholesterolemic rabbits. Mol Biol Int 1995; 37: 489-98.

27. Adelstein R, Ferguson LD, Rogers KA - Effects of dietary N-3 fatty acid supplementation on lipoproteins and intimal foam cell accumulation in the casein-fed rabbit. Clin Invest Med 1992; 15: 71-81.

28. Yano T, Mizuguchi K, Tanaka Y, Sato M - Effects of ethyl all-cis 5,8,14,17icosapentaenoate on low density lipoprotein in rabbits. Yakugaku-Zasshi 1995; 115: 843-51.

29. Phillipson BE, Rothorock DW, Connor WE, Harris WS, Illingworth DR - Reduction of plasma lipids, lipoproteins, and apoproteins by dietary fish oils in 
patients with hypertriglyceridemia. N Engl J Med 1985; 312: 1210.

30. Leaf A, Weber PC - Cardiovascular effects of n-3 fatty acids. NEngl J Med 1988; 318: 549 .

31. Sullivan DR, Sanders TAB, Trayner IM, Thompson GR-Paradoxical elevation of LDL apoprotein $B$ levels in hypertriglyceridaemic patients and normal subjects ingesting fish oil. Atherosclerosis 1986; 61: 129.

32. Shimokawa H, Lam JYT, Chesebro JH, Bowie EJW, Vanhoute PM-Effects of di- etary supplementation with cod-liver oil on endothelium-dependent response in porcine coronary arteries. Circulation 1987; 76: 898.

33. Kromhout D, Bosschieter EB, de Lezenne Coulander C - The inverse relation between fish consumption and 20-year mortality from coronary heart disease. $\mathrm{N}$ Engl J Med 1985; 312: 1205

34. Shekelle RB, Missell LV, Paul O, Stamler J - Fish consumption and mortality from coronary heart disease. N Engl J Med 1985; 313: 820. 